\title{
Independent associations between a metabolic syndrome severity score and future diabetes by sex and race: the Atherosclerosis Risk In Communities Study and Jackson Heart Study
}

\author{
Matthew J. Gurka ${ }^{1}$. Sherita H. Golden ${ }^{2,3}$ • Solomon K. Musani ${ }^{4}$ • Mario Sims ${ }^{4}$. \\ Abhishek Vishnu ${ }^{1,5} \cdot$ Yi Guo $^{1} \cdot$ Michelle Cardel $^{1}$. Thomas A. Pearson ${ }^{6}$. \\ Mark D. DeBoer ${ }^{7}$
}

Received: 20 December 2016/Accepted: 14 March 2017 /Published online: 4 April 2017

(C) Springer-Verlag Berlin Heidelberg 2017

\begin{abstract}
Aims/hypothesis The study aimed to assess for an association between the degree of severity of the metabolic syndrome and risk of type 2 diabetes beyond that conferred by the individual components of the metabolic syndrome.

Methods We assessed HRs for an Adult Treatment Panel III (ATP-III) metabolic syndrome score (ATP-III MetS) and a sex- and race-specific continuous metabolic syndrome severity $z$ score related to incident diabetes over a median of 7.8 years of follow-up among participants of two observational cohorts, the Atherosclerosis Risk in Communities study $(n=10,957)$ and the Jackson Heart Study $(n=2137)$.
\end{abstract}

Electronic supplementary material The online version of this article (doi:10.1007/s00125-017-4267-6) contains peer-reviewed but unedited supplementary material, which is available to authorised users.

Mark D. DeBoer

deboer@virginia.edu

1 Department of Health Outcomes and Policy, College of Medicine, University of Florida, Gainesville, FL, USA

2 Department of Medicine, Johns Hopkins University, Baltimore, MD, USA

3 Department of Epidemiology, Johns Hopkins University, Baltimore, MD, USA

4 Department of Medicine, Jackson Heart Study, University of Mississippi Medical Center, Jackson, MS, USA

5 Present address: Icahn School of Medicine at Mount Sinai, New York, NY, USA

6 Department of Epidemiology, College of Medicine, University of Florida, Gainesville, FL, USA

7 Department of Pediatrics, Division of Pediatric Endocrinology, University of Virginia, 409 Lane Road, Room 2017, PO Box 800386, Charlottesville, VA 22908, USA
Results The ATP-III MetS had an HR for incident diabetes of 4.36 (95\% CI 3.83, 4.97), which was attenuated in models that included the individual metabolic syndrome components. By contrast, participants in the fourth quartile of metabolic syndrome severity (compared with the first quartile) had an HR of 17.4 (95\% CI 12.6, 24.1) for future diabetes; in models that also included the individual metabolic syndrome components, this remained significant, with an HR of 3.69 (95\% CI 2.42, 5.64). There was a race $\times$ metabolic syndrome interaction in these models such that HR was greater for black participants (5.30) than white participants (2.24). When the change in metabolic syndrome severity score was included in the hazard models, this conferred a further association, with changes in metabolic syndrome severity score of $\geq 0.5$ having a HR of 2.66 compared with changes in metabolic syndrome severity score of $\leq 0$.

Conclusions/interpretation Use of a continuous sex- and race-specific metabolic syndrome severity $z$ score provided an additional prediction of risk of diabetes beyond that of the individual metabolic syndrome components, suggesting an added risk conferred by the processes underlying the metabolic syndrome. Increases in this score over time were associated with further risk, supporting the potential clinical utility of following metabolic syndrome severity over time.

Keywords Insulin resistance $\cdot$ Metabolic syndrome $\cdot$ Risk . Type 2 diabetes mellitus
Abbreviations
AIC
Akaike's information criterion
ARIC
Atherosclerosis Risk in Communities Study
ATP-III
Adult Treatment Panel III
ATP-III MetS 


$\begin{array}{ll}\text { CVD } & \text { Cardiovascular disease } \\ \text { JHS } & \text { Jackson Heart Study } \\ \text { ROC } & \text { Receiver operating characteristic } \\ \text { WC } & \text { Waist circumference }\end{array}$

\section{Introduction}

The cluster of cardiovascular risk factors known as the metabolic syndrome appears to be driven by underlying pathophysiological processes associated with insulin resistance, including adipocyte dysfunction, systemic inflammation, oxidative stress and disruption of cellular processes in target tissues [1-3]. Individuals classified as having the metabolic syndrome using traditional binary criteria such as those of the Adult Treatment Panel III (ATP-III) [4] have a high risk of disease progression, with an odds ratio of 5.16 for future type 2 diabetes [5]. Nevertheless, in most studies, a diagnosis of the metabolic syndrome does not confer additional predictive abilities beyond that of the individual metabolic syndrome components of elevated waist circumference (WC), high BP, high fasting triacylglycerol, low HDL-cholesterol and high fasting glucose [5]. Although abnormalities in these individual factors are not thought of as being causative of diabetes, researchers have questioned the value of the concept of the metabolic syndrome as an indication of the underlying pathophysiological processes (i.e. adipocyte dysfunction, systemic inflammation and oxidative stress) that themselves may contribute to the development of type 2 diabetes [6].

We developed a continuous metabolic syndrome severity $z$ score that is specific to sex and race/ethnicity [7-9]. This score correlates, among children and adults, with risk of future CVD [10] and diabetes [11]. However, these results were observed in a relatively small cohort of children originally enrolled over 40 years ago that did not allow for analyses by race or sex, nor did it allow for in-depth examination of the risk associated with the severity of the metabolic syndrome above and beyond the individual metabolic syndrome components. In a more recent combined cohort of adults, we demonstrated that this score remains a significant predictor of $\mathrm{CHD}$, even in models that include the individual metabolic syndrome components [12], suggesting that this score may capture additional risk conferred by biological processes underlying the metabolic syndrome [1].

The goal of this study was to assess the following: (1) the extent to which this continuous metabolic syndrome severity score was associated with future diabetes in a large, combined bi-racial cohort; (2) whether this score conferred additional risk prediction after accounting for each of the individual components of the metabolic syndrome; (3) whether the severity of the metabolic syndrome conferred differential risk by sex and race; (4) how the metabolic syndrome severity-related risk compared with the risk associated with ATP-III MetS; (5) whether a change in score over time conferred additional risk; and (6) how this risk compared with risk of CHD. We hypothesised that as a linear estimate of metabolic syndrome-related pathophysiology, this score would offer increased risk prediction for diabetes, both at baseline and over time, with potential relevance as a clinical tool.

\section{Methods}

\section{Cohorts and exclusion criteria}

This study and/or its analysis was approved by the Institutional Review Boards of the University of Florida, the University of Virginia and the study sites for the Atherosclerosis Risk in Communities Study (ARIC) and the Jackson Heart Study (JHS), and was carried out in accordance with the Declaration of Helsinki.

ARIC is a large community-based epidemiological cohort study that began in 1987-1989 across four field centres in the USA. Further details regarding the study design and objectives are published elsewhere [13]. ARIC data are available through ancillary study collaboration with ARIC [14]. A total of 15,792 primarily white and black participants aged 45-64 years old provided informed consent and were enrolled. We excluded individuals who did not consent $(n=395)$ and those who identified as a race/ethnicity other than white or black $(n=46)$. We used metabolic syndrome and risk factors from visits 1 and 2, and data on diabetes from visits $1-4$.

The JHS began as an extension study of black participants in the Jackson, MS, site of ARIC and used similar methodologies. JHS data are available through ancillary study collaboration with JHS [15]. Starting in 2000-2004, 5301 participants aged 21-95 years provided informed consent and were enrolled; this included 1626 participants who had been followed as part of ARIC and for whom data from ARIC rather than the JHS were used for the present analysis [16]. For JHS participants who did not participate in ARIC, we used metabolic syndrome data from visits 1 and 2, as well as diabetes data from visits $1-3$ (i.e. the final visit).

After combining the two cohorts as described above ( $n=19,026)$, we further excluded participants with baseline diabetes $(n=2485)$, CHD $(n=973)$ or stroke $(n=393)$, as well as participants who were missing baseline data on metabolic syndrome components $(n=792)$ or who had non-fasting laboratory results $(n=507)$, and/or those without follow-up data regarding outcomes $(n=2992)$. This left 13,094 individuals for our primary analysis. For this group of participants, we performed a subsequent analysis on those who had a follow-up visit at which the metabolic syndrome score could be calculated and who had yet to develop diabetes in order to estimate the association between change in metabolic syndrome severity and future diabetes. 


\section{Assessment of covariates and outcomes}

Exposure: the metabolic syndrome Components of the metabolic syndrome were measured using similar approaches for both cohorts as previously described [16, 17]. The metabolic syndrome was defined using the criteria established by the ATP-III, i.e. the presence of three or more of the following criteria: elevated WC ( $\geq 102 \mathrm{~cm}$ for men, $\geq 88 \mathrm{~cm}$ for women), elevated fasting triacylglycerol $(\geq 1.69 \mathrm{mmol} / \mathrm{l}[150 \mathrm{mg} / \mathrm{dl}])$, reduced HDL-cholesterol ( $<1.04 \mathrm{mmol} / \mathrm{l}$ [40 mg/dl] for men, $<1.29 \mathrm{mmol} / 1$ [50 mg/dl] for women), elevated BP ( $\geq 130 \mathrm{mmHg}$ systolic or $\geq 85 \mathrm{mmHg}$ diastolic, or drug treatment for hypertension) and elevated fasting blood glucose ( $\geq 5.55 \mathrm{mmol} / \mathrm{l}[100 \mathrm{mg} / \mathrm{dl}]$ ) [4].

Continuous metabolic syndrome severity $z$ scores at baseline were calculated for participants using sex- and race-based formulas. As described elsewhere [7, 8], these scores were derived using a confirmatory factor analysis approach for the five traditional metabolic syndrome components (WC, triacylglycerol, HDL-cholesterol, systolic BP, fasting glucose) to determine the weighted contribution of each component to a latent metabolic syndrome 'factor' on a sex- and race/ethnicity-specific basis. Confirmatory factor analysis was performed among adults aged 20-64 years from the National Health and Nutrition Examination Survey with categorisation into six subgroups based on sex and race/ethnicity (non-Hispanic white, nonHispanic black, Hispanic). For each of these six population subgroups, loading coefficients for the five metabolic syndrome components were transformed into a single metabolic syndrome factor and used to generate equations to calculate a standardised metabolic syndrome severity score for each subgroup (http:// mets.health-outcomes-policy.ufl.edu/calculator/, accessed 20 March 2017). The resulting metabolic syndrome severity scores are $z$ scores (normally distributed and ranging from theoretical negative to positive infinity with mean $=0$ and $\mathrm{SD}=1$ ) of relative metabolic syndrome severity on a sex- and race/ethnicity-specific basis. These scores correlate strongly with other markers of risk of the metabolic syndrome [18], including high-sensitivity C-reactive protein (hsCRP), uric acid and the homeostasis model of insulin resistance [8], with adiponectin [19] and with long-term risk of CVD [10, 12] and diabetes [11].

Outcome: type 2 diabetes In ARIC, participants were defined as having type 2 diabetes if they reported that a physician had told them they had diabetes, if they had a fasting serum glucose $\geq 6.99 \mathrm{mmol} / \mathrm{l}(126 \mathrm{mg} / \mathrm{dl})$ or a non-fasting serum glucose $\geq 11.10 \mathrm{mmol} / 1$ (200 mg/dl), or if they reported that they were taking insulin or oral hypoglycaemic medications [20, 21]. In the JHS, participants were defined as having diabetes if they had a fasting serum glucose $\geq 6.99 \mathrm{mmol} / 1(126 \mathrm{mg} / \mathrm{dl})$ or an $\mathrm{HbA}_{1 \mathrm{c}} \geq 6.5 \%(48 \mathrm{mmol} / \mathrm{mol})$ or if they had taken a diabetic medication within the 2 weeks before the clinic visit. These definitions of diabetes were used at visits $1-4$ for ARIC participants and at visits 1-3 for JHS participants. As the area of primary interest was the incidence of diabetes, time to diabetes was, for those individuals without diabetes at visit 1 , defined as the number of years between visit 1 and the first visit where diabetes was reported, regardless of diabetes status at subsequent visits.

\section{Statistics}

All statistical analyses were performed using SAS Version 9.4 (SAS Institute, Cary, NC, USA), and statistical significance was set to $\alpha=0.05$. To account for the interval censored timeto-event data (i.e. we do not know precisely when the diabetes developed), accelerated failure time models with an assumed Weibull distribution (using SAS PROC LIFEREG) were used, adjusting for baseline age and site (four sites from ARIC plus the JHS 'site'). HRs of interest were estimated as a function of the variables and the Weibull shape parameter estimate.

ATP-III MetS was modelled with and without its individual (binary) components to establish whether the metabolic syndrome was a risk factor above and beyond its components, similar to others [17]. Interactions were also examined to determine whether risk varied by sex and race. In a similar fashion, metabolic syndrome severity was modelled, using quartiles of metabolic syndrome severity (defined naturally by the associated percentiles since it is a $z$ score) and including quartiles for each of the metabolic syndrome components (defined by the analytical sample). This quartile approach allowed for an assessment of non-linear associations and threshold effects as well a reduction in the possibility of collinearity. Nonetheless, variance inflation factors were computed to assess the degree of collinearity when including the metabolic syndrome (or metabolic syndrome severity) and its components in the same model, with variance inflation factors greater than ten representing severe collinearity. Model fit was assessed and compared using Akaike's information criterion (AIC), with smaller values indicating a better fit. An unadjusted Kaplan-Meier estimate of survival by quartile of metabolic syndrome severity was performed, accounting for interval censored data (PROC ICLIFETEST). With a linear relationship observed between metabolic syndrome severity and risk of incident diabetes, a model with only the continuous score was fitted, and a time-dependent receiver operating characteristic (ROC) curve (and AUC) was generated [22] using a SAS macro. For those participants who had two metabolic syndrome scores calculated at visits 1 and 2, time to incident diabetes was modelled with both the visit 1 score and the change in score (categorised). Given that this was an evaluation of the risk of future diabetes using a metabolic syndrome score that included fasting glucose as a component, we also performed a sensitivity analysis using a metabolic syndrome severity score without glucose as one of the contributing components (i.e. incorporating only the remaining four metabolic syndrome components) for the association of baseline levels of this score with later diabetes. 


\section{Results}

\section{Participant characteristics and disease incidence}

We evaluated data from 13,094 participants meeting the inclusion criteria: 10,957 from ARIC and 2137 from the JHS. Participant characteristics at baseline by sex and race are shown in Table 1. At baseline, the prevalence of ATP-III MetS criteria was $31.2 \%$ and metabolic syndrome severity $z$ scores at baseline were on average 0 and were highest among white men.

Incident diabetes during a median of 7.8 years of follow-up was $12.2 \%$ for the entire cohort and differed significantly by sex and race, being highest among black women (17.1\%) and black men (16.8\%), intermediate among white men (11.7\%) and lowest among white women (8.0\%). Electronic supplementary material (ESM) Table 1 displays quartiles of metabolic syndrome severity score and diabetes incidence by ATP-III MetS status. Incident diabetes was higher among those with ATP-III MetS and higher by metabolic syndrome severity quartile, regardless of ATP-III MetS status.

\section{Survival models for the metabolic syndrome and incident diabetes}

Survival models for time to incident diabetes are shown in Table 2. ATP-III MetS was associated with future diabetes (HR 4.36, $p<0.01$ ), as were abnormalities in the individual components when assessed together in a model. When ATPIII MetS was assessed in a model with the individual metabolic syndrome components, the association between the ATP-III MetS and future diabetes was not significant.

Metabolic syndrome severity $z$ score (assessed as quartiles for the cohort) was also associated with future diabetes, with an HR for the highest vs lowest metabolic syndrome severity score quartile of $17.4(p<0.01)$ (Table 2). Each of the individual metabolic syndrome components was also associated with future diabetes when assessed in a model together. In a model that included metabolic syndrome severity $z$ score and the individual components, the metabolic syndrome $z$ score remained significantly associated with future diabetes, with an HR for the highest vs lowest quartile of $3.69(p<0.01)$. In our sensitivity analysis assessing a score that lacked glucose concentration as one of the metabolic syndrome components, we found an overall similar degree of association of that score with future diabetes as noted for the metabolic syndrome severity score, both with and without the individual metabolic syndrome components (including glucose) in the hazard model (ESM Table 2).

Table 3 shows HRs by sex and race for the metabolic syndrome predicting incident diabetes in models with and without adjustment for individual metabolic syndrome components. Models for ATP-III MetS unadjusted for individual components exhibited both race $\times$ metabolic syndrome and sex $\times$ metabolic syndrome interactions, with white women having the highest

Table 1 Descriptive characteristics of included participants

\begin{tabular}{|c|c|c|c|c|c|c|c|}
\hline \multirow[t]{2}{*}{ Characteristic } & \multirow[t]{2}{*}{ Overall } & \multicolumn{2}{|l|}{ Study } & \multicolumn{4}{|l|}{ Sex/race } \\
\hline & & ARIC & JHS & White men & White women & Black men & Black women \\
\hline $\mathrm{n}$ & 13,094 & 10,957 & 2137 & 3894 & 4710 & 1750 & 2740 \\
\hline \multicolumn{8}{|l|}{ Baseline means (SD) } \\
\hline Age (years) & $53.0(7.1)$ & $53.8(5.7)$ & $48.5(11.0)$ & $54.4(5.7)$ & $53.8(5.6)$ & $50.9(9.0)$ & $50.8(8.9)$ \\
\hline BMI & $27.8(5.7)$ & $27.1(5.0)$ & $31.5(7.2)$ & $27.2(3.8)$ & $26.1(5.0)$ & $28.3(5.4)$ & $31.4(7.2)$ \\
\hline $\mathrm{WC}(\mathrm{cm})$ & $95.9(13.7)$ & $95.4(13.2)$ & $98.4(16.1)$ & $98.9(10.0)$ & $91.5(13.6)$ & $97.4(13.8)$ & $98.1(16.3)$ \\
\hline Systolic BP (mmHg) & $120.0(17.5)$ & $119.5(17.6)$ & $122.4(16.5)$ & $119.2(15.3)$ & $116.0(17.2)$ & $126.4(18.4)$ & $123.6(18.3)$ \\
\hline HDL-cholesterol (mmol/l) & $1.36(0.43)$ & $1.37(0.44)$ & $1.32(0.35)$ & $1.13(0.32)$ & $1.53(0.44)$ & $1.26(0.39)$ & $1.48(0.42)$ \\
\hline Triacylglycerol (mmol/l) & $1.34(0.83)$ & $1.39(0.86)$ & $1.09(0.64)$ & $1.57(0.96)$ & $1.35(0.77)$ & $1.24(0.88)$ & $1.07(0.60)$ \\
\hline Glucose (mmol/l) & $5.38(0.54)$ & $5.47(0.51)$ & $4.94(0.47)$ & $5.59(0.49)$ & $5.36(0.48)$ & $5.28(0.57)$ & $5.17(0.58)$ \\
\hline MetS severity score & $0.0(0.8)$ & $0.1(0.8)$ & $-0.1(0.7)$ & $0.3(0.7)$ & $-0.1(0.8)$ & $-0.1(0.7)$ & $-0.0(0.8)$ \\
\hline \multicolumn{8}{|l|}{ Baseline rates, $\mathrm{n}(\%)$} \\
\hline ATP-III MetS & $4089(31.2 \%)$ & $3528(32.2 \%)$ & $561(26.3 \%)$ & $1362(35.0 \%)$ & $1341(28.5 \%)$ & $469(26.8 \%)$ & $917(33.5 \%)$ \\
\hline Blood pressure medication & $2784(22.2 \%)$ & $2074(19.0 \%)$ & $710(45.1 \%)$ & $586(15.1 \%)$ & $700(14.9 \%)$ & $502(34.1 \%)$ & $996(40.6 \%)$ \\
\hline Cholesterol medication & $2249(18.0 \%)$ & $2153(19.8 \%)$ & $96(6.1 \%)$ & $623(16.1 \%)$ & $915(19.5 \%)$ & $241(16.5 \%)$ & $470(19.2 \%)$ \\
\hline \multicolumn{8}{|l|}{ Incident diabetes $^{\mathrm{a}}$} \\
\hline Mean (SD) follow-up, years ${ }^{\mathrm{a}}$ & $7.8(2.0)$ & $7.8(2.1)$ & $7.5(1.4)$ & $7.9(2.1)$ & $8.1(1.9)$ & $7.3(2.0)$ & $7.4(2.0)$ \\
\hline $\mathrm{n}(\%)$ & $1595(12.2 \%)$ & $1246(11.4 \%)$ & $349(16.3 \%)$ & $457(11.7 \%)$ & $377(8.0 \%)$ & $294(16.8 \%)$ & $467(17.1 \%)$ \\
\hline
\end{tabular}

${ }^{\mathrm{a}}$ Based on date of last visit (subsequent modelling accounts for interval censoring)

MetS, metabolic syndrome 
Table 2 Survival models: time to incident diabetes

\begin{tabular}{|c|c|c|c|c|c|c|c|c|}
\hline \multirow[t]{2}{*}{ Model } & \multirow[t]{2}{*}{$\begin{array}{l}\text { Model baseline } \\
\text { covariates }\end{array}$} & \multirow[t]{2}{*}{$\begin{array}{l}\text { No. with } \\
\text { diabetes } \\
\text { (at risk) }\end{array}$} & \multicolumn{2}{|c|}{$\begin{array}{l}\text { ATP-III MetS criteria/MetS } \\
\text { Severity Score only }\end{array}$} & \multicolumn{2}{|c|}{$\begin{array}{l}\text { Risk factors }{ }^{\mathrm{a}} \text { (excluding } \\
\text { MetS criteria/Severity } \\
\text { Score) }\end{array}$} & \multicolumn{2}{|c|}{$\begin{array}{l}\text { Risk factors }{ }^{\mathrm{a}} \text { (with MetS } \\
\text { criteria/Severity Score) }\end{array}$} \\
\hline & & & HR $(95 \%$ CI) & $p$ value & HR $(95 \%$ CI $)$ & $p$ value & HR $(95 \%$ CI $)$ & $p$ value \\
\hline \multirow[t]{7}{*}{ ATP-III MetS models } & High WC & $1198(6660)$ & - & - & $2.18(1.93,2.48)$ & $<0.01$ & $2.21(1.93,2.55)$ & $<0.01$ \\
\hline & Low HDL-C & $850(4810)$ & - & - & $1.48(1.93,2.48)$ & $<0.01$ & $1.50(1.33,1.70)$ & $<0.01$ \\
\hline & High SBP & 931 (5309) & - & - & $1.36(1.33,1.65)$ & $<0.01$ & $1.38(1.21,1.56)$ & $<0.01$ \\
\hline & High TG & $573(2922)$ & - & - & $1.51(1.35,1.70)$ & $<0.01$ & $1.53(1.35,1.74)$ & $<0.01$ \\
\hline & High glucose & $1101(4769)$ & - & - & $4.98(4.30,5.78)$ & $<0.01$ & $5.05(4.30,5.94)$ & $<0.01$ \\
\hline & ATP-III MetS & $1014(4089)$ & $4.36(3.83,4.97)$ & $<0.01$ & - & - & $0.96(0.80,1.16)$ & 0.67 \\
\hline & Model AIC & & $11,539.59$ & & $10,582.11$ & & $10,583.93$ & \\
\hline \multirow{20}{*}{$\begin{array}{l}\text { MetS Severity } \\
\text { Score models }\end{array}$} & WC percentile ${ }^{\mathrm{b}}$ & & & & & & & \\
\hline & 25 th-50th & $310(3646)$ & - & - & $1.64(1.33,2.03)$ & $<0.01$ & $1.40(1.13,1.74)$ & $<0.01$ \\
\hline & 50th-75th & $462(3081)$ & - & - & $2.44(1.99,3.00)$ & $<0.01$ & $1.77(1.41,2.21)$ & $<0.01$ \\
\hline & $>75$ th & $693(2915)$ & - & - & $3.47(2.82,4.27)$ & $<0.01$ & $2.13(1.68,2.70)$ & $<0.01$ \\
\hline & HDL-C percentile ${ }^{b}$ & & & & & & & \\
\hline & 25 th-50th & $561(4054)$ & - & - & $0.90(0.80,1.01)$ & 0.07 & $0.97(0.86,1.10)$ & 0.63 \\
\hline & 50th-75th & 259 (2876) & - & - & $0.73(0.63,0.86)$ & $<0.01$ & $0.87(0.74,1.02)$ & 0.09 \\
\hline & $>75$ th & $161(2816)$ & - & - & $0.55(0.45,0.67)$ & $<0.01$ & $0.75(0.61,0.92)$ & $<0.01$ \\
\hline & SBP percentile ${ }^{b}$ & & & & & & & \\
\hline & 25 th- -50 th & 419 (3865) & - & - & $1.17(1.00,1.38)$ & 0.05 & $1.12(0.95,1.32)$ & 0.17 \\
\hline & 50th-75th & 445 (2905) & - & - & $1.45(1.23,1.71)$ & $<0.01$ & $1.34(1.14,1.58)$ & $<0.01$ \\
\hline & $>75$ th & 497 (2928) & - & - & $1.43(1.21,1.69)$ & $<0.01$ & $1.24(1.05,1.47)$ & 0.01 \\
\hline & TG percentile ${ }^{\mathrm{b}}$ & & & & & & & \\
\hline & 25 th-50th & $322(3426)$ & - & - & $0.92(0.78,1.10)$ & 0.34 & $0.80(0.66,0.95)$ & 0.01 \\
\hline & 50th-75th & $400(3113)$ & - & - & $1.09(0.92,1.29)$ & 0.37 & $0.82(0.68,1.00)$ & 0.05 \\
\hline & $>75$ th & $650(3166)$ & - & - & $1.34(1.13,1.58)$ & $<0.01$ & $0.90(0.74,1.09)$ & 0.29 \\
\hline & Glucose percentile $^{\mathrm{b}}$ & & & & & & & \\
\hline & 25th-50th & $207(3691)$ & - & - & $1.29(1.04,1.60)$ & 0.02 & $1.18(0.95,1.46)$ & 0.13 \\
\hline & 50th-75th & 309 (2937) & - & - & $2.49(2.02,3.06)$ & $<0.01$ & $2.11(1.70,2.60)$ & $<0.01$ \\
\hline & $>75$ th & $932(3023)$ & - & - & $8.16(6.56,10.17)$ & $<0.01$ & $6.38(5.11,7.95)$ & $<0.01$ \\
\hline \multirow{5}{*}{$\begin{array}{l}\text { MetS Severity } \\
\text { Score overall }\end{array}$} & MetS score percentile & & & & & & & \\
\hline & 25 th-50th & $211(3993)$ & $2.51(1.84,3.43)$ & $<0.01$ & - & - & $1.50(1.07,2.11)$ & $<0.01$ \\
\hline & 50 th-75th & $539(4008)$ & $6.93(5.10,9.42)$ & $<0.01$ & - & - & $2.40(1.65,3.50)$ & $<0.01$ \\
\hline & $>75$ th & 794 (2731) & $17.39(12.57,24.05)$ & $<0.01$ & - & - & $3.69(2.42,5.64)$ & $<0.01$ \\
\hline & Model AIC & & $11,199.02$ & & $10,503.75$ & & $10,452.07$ & \\
\hline
\end{tabular}

All models adjusted for baseline age and site/centre.

${ }^{a}$ MetS components were included together in the models as dichotomous variables when comparing with or run in a model with ATP-III MetS (top portion of table) and as quartiles when comparing with or run in a model with MetS severity score (bottom portion of table)

${ }^{\mathrm{b}}$ Reference category $<25$ th percentile

Glu, glucose; HDL-C, HDL-cholesterol; MetS, metabolic syndrome; pctile, percentile; SBP, systolic blood pressure; Sev, severity; TG, triacylglycerol

association (HR 6.28) and black men the lowest association (HR 2.88). Following adjustment for the individual components, no interaction remained, with a non-significant protective association of ATP-III MetS among all participants $(\mathrm{HR}=0.95$, $p=0.594)$.

Assessing correlations between the metabolic syndrome severity score and future diabetes in models unadjusted for the individual metabolic syndrome components, there was a sex $\times$ race $\times$ metabolic syndrome interaction, with black women showing the strongest association (HR for highest vs lowest quartile 33.6) and white men having the weakest association (HR 6.48) (Table 3). In a model that included the individual components, there was only a race $\times$ metabolic syndrome interaction, with black men and women having a higher 
Table 3 Survival models: time to incident diabetes

\begin{tabular}{|c|c|c|c|c|c|}
\hline \multirow[t]{2}{*}{ Model including: } & \multicolumn{4}{|c|}{ Sex and race-specific $\mathrm{HR}^{\mathrm{a}}$} & \multirow{2}{*}{$\begin{array}{l}\text { Significant MetS interactions } \\
(p<0.05)\end{array}$} \\
\hline & White men & White women & Black men & Black women & \\
\hline \multicolumn{6}{|l|}{ ATP-III MetS } \\
\hline Unadjusted & $4.71(3.88,5.73)$ & $6.28(5.08,7.78)$ & $2.88(2.36,3.52)$ & $3.85(3.20,4.63)$ & Sex $\times$ MetS, race $\times$ MetS \\
\hline $\begin{array}{l}\text { Adjusted for individual } \\
\text { components }{ }^{\mathrm{b}}\end{array}$ & $0.95(0.79,1.15)$ & $0.95(0.79,1.15)$ & $0.95(0.79,1.15)$ & $0.95(0.79,1.15)$ & None \\
\hline \multicolumn{6}{|l|}{ MetS severity percentile ${ }^{c}$} \\
\hline Unadjusted & & & & & Sex $\times$ race $\times$ MetS \\
\hline 25 th-50th & $1.36(0.76,2.41)$ & $2.15(1.19,3.87)$ & $1.84(0.99,3.42)$ & $5.68(2.72,11.88)$ & \\
\hline 50th-75th & $2.17(1.27,3.72)$ & $6.64(3.82,11.55)$ & $6.67(3.74,11.89)$ & $17.08(8.27,35.32)$ & \\
\hline$>75$ th & $6.48(3.81,11.00)$ & $25.51(14.67,44.38)$ & $11.91(6.58,21.56)$ & $33.56(16.12,69.88)$ & \\
\hline $\begin{array}{l}\text { Adjusted for individual } \\
\text { components }\end{array}$ & & & & & Race $\times$ MetS \\
\hline 25 th-50th & $0.97(0.62,1.53)$ & $0.97(0.62,1.53)$ & $2.15(1.28,3.62)$ & $2.15(1.28,3.62)$ & \\
\hline 50 th-75th & $1.29(0.76,2.19)$ & $1.29(0.76,2.19)$ & $4.00(2.22,7.18)$ & $4.00(2.22,7.18)$ & \\
\hline$>75$ th & $2.24(1.21,4.15)$ & $2.24(1.21,4.15)$ & $5.30(2.73,10.29)$ & $5.30(2.73,10.29)$ & \\
\hline
\end{tabular}

Primary predictor: MetS (ATP-III or MetS $z$ score), overall and by sex and race.

${ }^{a}$ Adjusted for site/centre baseline age, sex and race (and sex $\times$ race interaction)

${ }^{\mathrm{b}}$ MetS components were included together in the models as dichotomous variables when evaluating ATP-III MetS, and as quartiles when evaluating MetS severity score

${ }^{\mathbf{c}}$ Reference category $<25$ th percentile

MetS, metabolic syndrome

degree of association (HR for highest vs lowest quartile 5.30) than white men and women (HR 2.24).

\section{Kaplan-Meier plot of time to disease by metabolic syndrome severity quartile}

Figure 1 displays Kaplan-Meier plots of time to incident diabetes by quartile of metabolic syndrome severity over a 10 year period. There was an increase in probability of 10 year diabetes-free survival ranging from $96 \%$ for the first quartile to $60 \%$ for the fourth quartile.

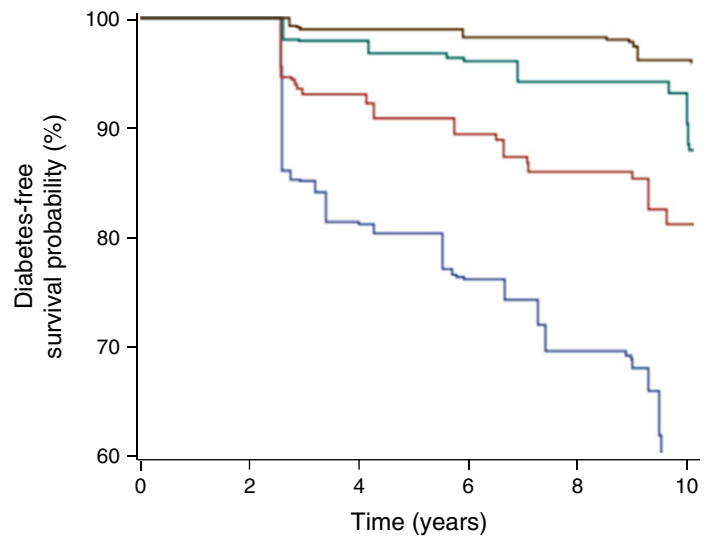

Fig. 1 Kaplan-Meier curve of diabetes-free survival by metabolic syndrome severity quartile. Baseline metabolic syndrome percentile: brown line, <25th; green line, 25th-50th; red line, 50th-75th; blue line, $>75$ th

\section{ROC curves, sensitivity and specificity}

ESM Fig. 1 shows a ROC curve for 10 year risk of diabetes by baseline metabolic syndrome $z$ score. The AUC for metabolic syndrome severity as a predictor of diabetes was 0.77 . This compared with a $\mathrm{c}$ statistic from logistic regression of 0.68 for ATP-III MetS. The cut-off of metabolic syndrome $z$ score that maximised sensitivity and specificity for incident diabetes was 0.25 , which had a sensitivity of 0.68 and a specificity of 0.72 (ESM Table 3). This compared with ATP-III MetS, which had a sensitivity of 0.64 and specificity of 0.73 for predicting diabetes.

\section{Change in metabolic syndrome severity over time and incident diabetes}

Metabolic syndrome $z$ scores were available for 11,292 individuals at both visits 1 and 2 , with a mean $( \pm \mathrm{SD})$ change in $z$ score of $0.2( \pm 0.4)$ units over $3.2( \pm 0.7)$ years. Of these, 700 (6.2\%) individuals went on to develop diabetes over a mean 5.1 $( \pm 1.4)$ years after visit 2 . Table 4 shows the results of a model that included metabolic syndrome severity at visit 1 and change in $z$ score between visits 1 and 2. Compared with individuals with a change in score $<0$, participants with a change $>0.5$ had a HR of 2.66 for incident diabetes $(p<0.001)$, independent of their visit 1 score. 
Table 4 Survival model of time to incident diabetes after two clinic visits $(n=11,292)$

\begin{tabular}{|c|c|c|}
\hline Covariate & Estimated HR (95\% CI) & $p$ value \\
\hline \multicolumn{3}{|l|}{ Model 1: visit 1 only } \\
\hline \multicolumn{3}{|c|}{ Visit 1 MetS percentile range $(z \text { score range })^{\mathrm{a}}$} \\
\hline 25 th-50th $(-0.675$ to 0$)$ & $2.43(1.55,3.79)$ & $<0.001$ \\
\hline 50 th -75 th $(0$ to 0.675$)$ & $6.10(3.90,9.52)$ & $<0.001$ \\
\hline$>75$ th $(>0.675)$ & $15.93(9.86,25.73)$ & $<0.001$ \\
\hline \multicolumn{3}{|l|}{$\mathrm{AIC}=5489.14$} \\
\hline \multicolumn{3}{|l|}{ Model 2: visit 1 and visit 2} \\
\hline \multicolumn{3}{|c|}{ Visit 1 MetS percentile range $(z \text { score range })^{\mathrm{a}}$} \\
\hline 25 th -50 th $(-0.675$ to 0$)$ & $2.52(1.62,3.95)$ & $<0.001$ \\
\hline 50 th -75 th $(0$ to 0.675$)$ & $6.87(4.38,10.78)$ & $<0.001$ \\
\hline$>75$ th $(>0.675)$ & $20.00(12.22,32.73)$ & $<0.001$ \\
\hline \multicolumn{3}{|c|}{ Change in MetS $z$ score (visit $2-$ visit 1$)^{\mathrm{b}}$} \\
\hline $0-0.25$ & $1.20(0.96,1.49)$ & 0.104 \\
\hline $0.25-0.50$ & $1.87(1.50,2.31)$ & $<0.001$ \\
\hline$>0.50$ & $2.66(2.13,3.32)$ & $<0.001$ \\
\hline $\mathrm{AIC}=5394.7$ & & \\
\hline
\end{tabular}

Data are adjusted for site/centre and baseline age.

${ }^{a}$ Reference category $<25$ th percentile

${ }^{\mathrm{b}}$ Reference change category $<0$

MetS, metabolic syndrome

\section{Discussion}

Although the abnormalities in CVD risk factors that comprise the metabolic syndrome have long been known to be associated with insulin resistance and metabolic dysfunction, the majority of prior studies have not found that the presence of the metabolic syndrome provided additional predictive power for future disease beyond the metabolic syndrome components themselves [5]. In the current study of a large, combined bi-racial cohort, we present novel findings: (1) that a continuous metabolic syndrome severity $z$ score remained strongly associated with future diabetes, even in models that included the individual components of the metabolic syndrome; (2) that this association varied by race, with a higher risk among black participants; and (3) that the degree of change in metabolic syndrome score over a 4 year period conferred the additional risk. This study expands on our previous similar findings with respect to CHD [12] and our findings regarding risk of diabetes in a small, regional cohort [11]. These findings are consistent with the hypothesis that a higher degree of metabolic syndrome severity may contribute to future risk of diabetes, potentially as an estimate of the underlying metabolic dysfunction driving the abnormalities in the individual metabolic syndrome components [2,3]. Furthermore, the heightened risk associated with the change in metabolic syndrome score over time suggests the potential to follow such a score over time (potentially provided automatically using an electronic medical record calculator) for ominous worsening of risk, as well potentially to motivate patients [3] and gauge the response to therapies [23].

It should be noted that adjustment for the individual metabolic syndrome components has a more logical basis in considering risk for CHD than for type 2 diabetes. This is because the suggestion has been that if risk of CHD could be reduced by merely addressing the abnormalities in the individual components (i.e. lowering triacylglycerol levels, decreasing BP and raising HDL-cholesterol), there might not be as much utility in knowing about the underlying processes that drive these abnormalities [6]. The case of type 2 diabetes is, however, different in that these individual components-while potentially linked by adipocyte dysfunction, systemic inflammation and oxidative stress - are not likely themselves to be individual drivers of diabetes. Nevertheless, the fact that the severity of the metabolic syndrome persists in its association with future diabetes in models that include each of these abnormalities suggests that the strength of their association together is itself a potentially important estimate of risk.

Prior to adjustment for the individual metabolic syndrome components, the metabolic syndrome severity-related risk for diabetes was threefold higher among women than men, and $30-80 \%$ higher among black than white participants. This occurred despite the differential weights of the metabolic syndrome components determined by sex and race to account for how the metabolic syndrome was manifested among each group. We had not previously noted interactions of sex or race with metabolic syndrome severity in the prediction of future CHD [12], suggesting that the sex- and race-specificity of the score may have estimated metabolic syndrome-related CHD risk similarly for all groups. The higher association of metabolic syndrome severity and diabetes risk among women (prior to adjustment for metabolic syndrome components) may reflect an ability to better separate individuals with 'healthy' obesity [24] compared with those with higher metabolic risk. Before adjustment for the individual metabolic syndrome components, women in the fourth quartile of metabolic syndrome severity (compared with the first quartile) had HRs of 25.5 and 33.6 for white and black women, respectively, emphasising the potential utility of such a score for risk identification that is more powerful than that of ATP-III MetS, which conferred HRs of 6.3 and 3.6. This sex $\times$ race $\times$ metabolic syndrome severity interaction was not present when the individual components of the metabolic syndrome were included in the model, providing further support that the elevated metabolic syndrome severity-related risk for women may be related to abnormalities in specific metabolic syndrome components. Nevertheless, metabolic syndrome severity continued to add predictive ability for future diabetes among white and black women in models adjusted for the components, supporting additional risk related to the processes driving the metabolic syndrome. 
The differential metabolic syndrome-related risk between races had not previously been observed, possibly because earlier studies had fewer black participants [5, 25]. Indeed, it has been noted that the metabolic syndrome did not provide adequate risk prediction among black participants [26, 27], who have a relatively low prevalence of the metabolic syndrome as assessed using traditional binary criteria despite having high rates of diabetes [28] and CHD [29], as though the metabolic syndrome were failing to detect some degree of risk, particularly among black men [30-33]. It is partly for this reason that we formulated scores in an attempt to reflect how the metabolic syndrome is manifested on a sex- and race/ethnicityspecific basis [8, 34]. Although population-based metabolic syndrome severity was similar at baseline by sex and race, the higher metabolic syndrome-related risk of diabetes among black participants suggests an important interplay between metabolic syndrome severity and other factors contributing to diabetes progression.

Overall metabolic syndrome severity in these combined cohorts was less associated with risk of CHD [12] than of diabetes, as has previously been seen with respect to ATP-III MetS [5, 35]. In models of future disease prediction without the individual metabolic syndrome components, the fourth quartile of metabolic syndrome severity (compared with the first) had an HR of 4.0 for CHD and of 17.4 for future diabetes. This appears to emphasise that a greater degree of insulin resistance (potentially contributing to earlier beta cell exhaustion) and other processes associated with the metabolic syndrome are more directly related to diabetes risk than to risk of atherosclerosis per se. Nevertheless, for both disease outcomes, metabolic syndrome severity remained a significant risk factor, even when the individual metabolic syndrome components were included - and in a sensitivity analysis, metabolic syndrome severity was associated with risk of future diabetes, even when using a metabolic syndrome score without glucose concentration as a component.

Although a continuous score such as this provides many benefits, including improvements in power for statistical analysis and the ability to track changes over time, clinical application would probably benefit from cut-off values to identify individuals at highest risk. We found that a metabolic syndrome severity score cut-off of 0.25 provided reasonable sensitivity $(0.68)$ and specificity $(0.72)$ for future diabetes, although clearly such a cut-off could be adjusted to emphasise the detection of a larger number of individuals at risk or to try to maximise specificity. Overall, this was similar to the sensitivity and specificity for ATP-III MetS of 0.64 and 0.73 , but with the added benefit of being able to track for changes in risk over time. We had not noted an obvious cut-off in the association between metabolic syndrome severity and CHD risk [12], and ultimately different cut-offs may be needed for different disease states.

This study had many limitations. We relied on identification of a diagnosis of diabetes at study visits, whereas the actual development of disease probably occurred at intermediate time points. In addition, the definition of type 2 diabetes differed between the two cohorts, with the JHS including elevated $\mathrm{HbA}_{1 \mathrm{c}}$ as an indicator of diabetes; this would potentially identify more individuals who had previously unidentified diabetes but may have had normal fasting blood glucose levels. Because JHS participants made up $48 \%$ of the black participants analysed, there is the possibility that a higher identification of diabetes in this cohort could have affected the differences in ability of the metabolic syndrome severity score to predict diabetes by race/ethnicity. Participants were followed through the 1990s (ARIC) and 2000s (JHS), and interval changes in health maintenance may limit the generalisability to current populations. Nevertheless, participants from ARIC and other cohorts from the 1990s were also used in deriving the recent American College of Cardiology/ American Heart Association risk prediction algorithm cohorts for risk of CHD, supporting the idea that population-based risk has not changed significantly [36]. This study had several strengths in the longitudinal follow-up of two large cohorts, with a significant representation of white and black, and male and female participants.

In conclusion, we found that a sex- and race/ethnicity-specific continuous metabolic syndrome $z$ score was associated with risk of future diabetes in models that included each of the individual metabolic syndrome components. A score such as this may thus provide additional risk prediction related to the underlying processes that drive the clustering of abnormalities among metabolic syndrome components. Temporal increases in the score escalated risk, supporting the potential to track score levels using electronic medical records to trigger additional therapies and follow treatment response over time.

Acknowledgements The authors thank the staff and participants of the ARIC study and JHS for their important contributions.

Data availability These data are available through ancillary studies with the Atherosclerosis Risk in Communities Study (https://www2. cscc.unc.edu/aric/) and the Jackson Heart Study (https://www. jacksonheartstudy.org/).

Funding This work was supported by NIH grants 1R01HL120960 (MJG and MDD). The JHS is supported by contracts HHSN268201300046C, HHSN268201300047C, HHSN268201300048C, HHSN268201300049C and HHSN268201300050C from the National Heart, Lung, and Blood Institute and the National Institute on Minority Health and Health Disparities. The ARIC Study is carried out as a collaborative study supported by National Heart, Lung, and Blood Institute contracts (HHSN268201100005C, HHSN268201100006C, HHSN268201100007C, HHSN268201100008C, HHSN268201100009C, HHSN268201100010C, HHSN268201100011C and HHSN268201100012C).

Duality of interest The authors declare that there is no duality of interest associated with this manuscript. 
Contribution statement MJG participated in the design, analysis, interpretation and write-up of the research. SHG and MC participated in the interpretation of the research and editing of the manuscript. SKM and MS participated in the design and interpretation of the research, and editing of the manuscript. AV, YG and TAP participated in the design, analysis and interpretation of the research, and editing of the manuscript. MDD participated in the design and analysis, was responsible for the write-up and had primary responsibility for the final content. All authors gave final approval of the version to be published.

\section{References}

1. Shulman GI (2014) Ectopic fat in insulin resistance, dyslipidemia, and cardiometabolic disease. N Engl J Med 371:2237-2238

2. de Ferranti S, Mozaffarian D (2008) The perfect storm: obesity, adipocyte dysfunction, and metabolic consequences. Clin Chem 54:945-955

3. DeBoer MD (2013) Obesity, systemic inflammation, and increased risk for cardiovascular disease and diabetes among adolescents: a need for screening tools to target interventions. Nutrition 29:379386

4. Grundy SM, Cleeman JI, Daniels SR et al (2005) Diagnosis and management of the metabolic syndrome - an American Heart Association/National Heart, Lung, and Blood Institute Scientific Statement. Circulation 112:2735-2752

5. Ford ES, Li C, Sattar N (2008) Metabolic syndrome and incident diabetes: current state of the evidence. Diabetes Care 31:1898-1904

6. Kahn R, Buse J, Ferrannini E, Stern M (2005) The metabolic syndrome: time for a critical appraisal: joint statement from the American Diabetes Association and the European Association for the Study of Diabetes. Diabetes Care 28:2289-2304

7. Gurka MJ, Ice CL, Sun SS, DeBoer MD (2012) A confirmatory factor analysis of the metabolic syndrome in adolescents: an examination of sex and racial/ethnic differences. Cardiovasc Diabetol 11: 128

8. Gurka MJ, Lilly CL, Norman OM, DeBoer MD (2014) An examination of sex and racial/ethnic differences in the metabolic syndrome among adults: a confirmatory factor analysis and a resulting continuous severity score. Metabolism 63:218-225

9. Lee AM, Gurka MJ, DeBoer MD (2016) A metabolic syndrome severity score to estimate risk in adolescents and adults: current evidence and future potential. Expert Rev Cardiovasc Ther 14: 411-413

10. DeBoer MD, Gurka MJ, Woo JG, Morrison JA (2015) Severity of metabolic syndrome as a predictor of cardiovascular disease between childhood and adulthood: the Princeton Lipid Research Cohort Study. J Am Coll Cardiol 66:755-757

11. DeBoer MD, Gurka MJ, Woo JG, Morrison JA (2015) Severity of the metabolic syndrome as a predictor of type 2 diabetes between childhood and adulthood: the Princeton Lipid Research Cohort Study. Diabetologia 58:2745-2752

12. DeBoer MD, Gurka MJ, Hill Golden S et al (2016) Independent associations between metabolic syndrome severity and future coronary heart disease by sex and race. J Am Coll Cardiol 69:12041205

13. The ARIC Investigators (1989) The Atherosclerosis Risk in Communities (ARIC) Study: design and objectives. Am J Epidemiol 129: 687-702

14. Collaborative Studies Coordinating Center (2017) Atherosclerosis Risk in Communities Study Description. Available from: https:// www2.cscc.unc.edu/aric/. Accessed 20 Mar 2017

15. Jackson Heart Study Investigators (2015) Welcome to the Jackson Heart Study Home Page. Available from https://www.
jacksonheartstudy.org/Research/Ancillary-Studies. Accessed 20 Mar 2017

16. Taylor HA, Wilson JG, Jones DW et al (2005) Toward resolution of cardiovascular health disparities in African Americans: design and methods of the Jackson Heart Study. Ethn Dis 15:S6-4-17

17. McNeill AM, Schmidt MI, Rosamond WD et al (2005) The metabolic syndrome and 11-year risk of incident cardiovascular disease in the atherosclerosis risk in communities study. Diabetes Care 28: 385-390

18. DeBoer MD, Gurka MJ (2010) Ability among adolescents for the metabolic syndrome to predict elevations in factors associated with type 2 diabetes and cardiovascular disease: data from the national health and nutrition examination survey 1999-2006. Metab Syndr Relat Disord 8:343-353

19. DeBoer MD, Gurka MJ, Morrison JA, Woo JG (2016) Interrelationships between the severity of metabolic syndrome, insulin and adiponectin and their relationship to future type 2 diabetes and cardiovascular disease. Int J Obes 40:1353-1359

20. Schmidt MI, Duncan BB, Bang H et al (2005) Identifying individuals at high risk for diabetes: the Atherosclerosis Risk in Communities study. Diabetes Care 28:2013-2018

21. Effoe VS, Correa A, Chen H, Lacy ME, Bertoni AG (2015) Highsensitivity C-reactive protein is associated with incident type 2 diabetes among African Americans: the Jackson Heart Study. Diabetes Care 38:1694-1700

22. Heagerty PJ, Lumley T, Pepe MS (2000) Time-dependent ROC curves for censored survival data and a diagnostic marker. Biometrics 56:337-344

23. Vishnu A, Gurka MJ, DeBoer MD (2015) The severity of the metabolic syndrome increases over time within individuals, independent of baseline metabolic syndrome status and medication use: the Atherosclerosis Risk in Communities Study. Atherosclerosis 243: 278-285

24. Aguilar-Salinas CA, Garcia EG, Robles L et al (2008) High adiponectin concentrations are associated with the metabolically healthy obese phenotype. J Clin Endocrinol Metab 93:4075-4079

25. Hanley AJ, Karter AJ, Williams K et al (2005) Prediction of type 2 diabetes mellitus with alternative definitions of the metabolic syndrome: the Insulin Resistance Atherosclerosis Study. Circulation 112:3713-3721

26. Sumner AE (2009) Ethnic differences in triglyceride levels and high-density lipoprotein lead to underdiagnosis of the metabolic syndrome in black children and adults. J Pediatr 155:e7-e11

27. DeBoer MD (2010) Underdiagnosis of metabolic syndrome in nonHispanic black adolescents: a call for ethnic-specific criteria. Curr Cardiovasc Risk Rep 4:302-310

28. Cowie CC, Rust KF, Byrd-Holt DD et al (2010) Prevalence of diabetes and high risk for diabetes using A1C criteria in the U.S. population in 1988-2006. Diabetes Care 33:562-568

29. Mozaffarian D, Benjamin EJ, Go AS et al (2016) Heart disease and stroke statistics - 2016 update: a report from the American Heart Association. Circulation 133:e38-e360

30. Walker SE, Gurka MJ, Oliver MN, Johns DW, DeBoer MD (2012) Racial/ethnic discrepancies in the metabolic syndrome begin in childhood and persist after adjustment for environmental factors. Nutr Metab Cardiovasc Dis 22:141-148

31. DeBoer MD, Gurka MJ, Sumner AE (2011) Diagnosis of the metabolic syndrome is associated with disproportionately high levels of high-sensitivity C-reactive protein in non-Hispanic black adolescents: an analysis of NHANES 1999-2008. Diabetes Care 34: 734-740

32. DeBoer MD, Dong L, Gurka MJ (2011) Racial/ethnic and sex differences in the ability of metabolic syndrome criteria to predict elevations in fasting insulin levels in adolescents. J Pediatr 159: 975-981 
33. DeBoer MD, Dong L, Gurka MJ (2012) Racial/ethnic and sex differences in the relationship between uric acid and metabolic syndrome in adolescents: an analysis of National Health and Nutrition Survey 1999-2006. Metabolism 61:554-561

34. DeBoer MD (2011) Ethnicity, obesity and the metabolic syndrome: implications on assessing risk and targeting intervention. Expert Rev Endocrinol Metab 6:279-289
35. Mottillo S, Filion KB, Genest J et al (2010) The metabolic syndrome and cardiovascular risk: a systematic review and metaanalysis. J Am Coll Cardiol 56:1113-1132

36. Goff DC, Lloyd-Jones DM, Bennett G et al (2014) 2013 ACC/AHA guideline on the assessment of cardiovascular risk: a report of the American College of Cardiology/American Heart Association Task Force on Practice Guidelines. Circulation 129:S49-S73 